\title{
A Framework for Multi-Threshold Image Segmentation of Low Contrast Medical Images
}

\author{
Ahmed Elaraby $^{1 *}$, Ismail Elansary ${ }^{2}$ \\ ${ }^{1}$ Department of Computer Science, Faculty of Computers and Information, South Valley University, Qena 83523, Egypt \\ ${ }^{2}$ Modern Academy for Computer Science and Management Technology, Cairo 11742, Egypt
}

Corresponding Author Email: ahmed.elaraby@svu.edu.eg

https://doi.org/10.18280/ts.380207

Received: 15 December 2020

Accepted: 20 March 2021

\section{Keywords:}

medical image, segmentation, fuzzy hill entropy, differential evolution

\begin{abstract}
Accurate medical images segmentation plays a vital role in contouring during diagnosis and treatment planning. To improve the segmentation accuracy in low contrast images, we propose a method by combining Hill entropy and fuzzy c-partition. Here, using membership function, an image is first transformed into fuzzy domain. Subsequently, the fuzzy Hill entropies are defined for foreground (object) and background. Next, the total fuzzy Hill entropy is maximized to compute the accurate threshold; this process is employed to calculate a proper parameter combination of membership function. This Hill entropy is then optimized to acquire an image threshold by Differential Evolution "DE" optimization algorithm. The key benefit of the presented approach is that it considers the information of background and object as well as interactions between them in threshold selection mechanism. The results and performance evaluations show the better accuracy of our technique over other existing approaches.
\end{abstract}

\section{INTRODUCTION}

Image segmentation is employed to extract the specific object from the image with the help of extracted features from the dataset. Here, features are used to show the similar and different areas. Medical image segmentation is one the complex task due to numerous challenges like blurring, distortion, low contrast, and low resolution. Lot of techniques has been developed for segmenting the medical image. It contains transformation approaches like watershed segmentation, texture and threshold methods like entropy based and Otsu's approach.

Extensive studies have been conducted for segmenting the medical image. Fuzzy set theory and information theory have a huge impact on image segmentation [1-3]. Fuzzy entropy has become one of the important research points for threshold based medical image segmentation. Zhao et al. [4] proposed a technique to compute the compatibility of the "fuzzy cpartition" and the probability partition. In the further study, three level membership functions such as S-function, $\pi$ function, and Z-function are used by Tao et al. [5]. The entropy concept from the information theory is used for threshold purpose. Furthermore, Kapur et al. [6] enhanced this technique through histogram of image based maximum fuzzy entropy approach. Here entropy of foreground as well as background has been maximized. However, this technique is affected by the noise evidently due to one dimensional histogram. To remove above shortcoming and make full benefit of pixel intensity, space information and neighborhood pixels; two dimensional histograms has been employed for image segmentation [7, 8]. Sengar and Mukhopadhyay [9] presented an approach for segmentation with the help of normalized selfadaptive optical flow. Otsu's approach has been employed in this method to select the threshold parameter $[10,11]$. The greyness and spatial uncertainties among pixels are handled through fuzzy homogeneity vectors and multilevel threshold [12]. To improve the performance, "Three level image segmentation using non extensive entropy" is proposed by EIFegh et al. [13]. Active contour models have been learned for medical image segmentation [14]. Cross entropy and dice entropy have been employed for segmentation problems [15]. Level set functions are used to construct the segmentation model, it is an energy minimization problem, which is solved through "partial differential equations". Furthermore, this model has been enhanced to texture and multiphase problems $[16,17]$

Lots of approaches have been proposed to accurately segment the medical image. However, still there are some deficiencies in the existing algorithms specially related to low contrast and resolution. To address the issue of low contrast image, we proposed an efficient medical image segmentation method by combining "Hill entropy and fuzzy c-partition" [18]. First, we transferred image into fuzzy domain with the help of membership function. Subsequently, the fuzzy hill entropies have been defined for foreground and background. Next, threshold is computed through maximizing the total fuzzy Hill entropy. Furthermore, differential evolution optimization technique is applied to optimize Hill entropy measure [19]. Our technique has been tested on different medical images that show efficient and accurate results.

The remaining part of this article is structured as follows; proposed technique is elaborated in Section 2. Experimental results and performance evaluation are provided in Section 3. Finally, Section 4 presents the conclusion.

\section{PROPOSED APPROACH}

Threshold techniques based on entropy have attracted a considerable attention over the last few years in image 
processing. They have been found to be among the most powerful techniques in image segmentation. For this reason, we propose here a framework approach that combining Hill entropy and fuzzy c-partition for medical image segmentation.

The Shannon entropy [20] is defined as:

$$
H(p)=-\sum_{i=1}^{k} p_{i} \ln \left(p_{i}\right)
$$

The generalized entropy of Hill [20] is defined as:

$$
N_{\alpha}=\left(\sum_{i=1}^{W} p_{i}^{\alpha}\right)^{\frac{1}{1-\alpha}}, \text { for } \alpha \geq 0 \text { and } \alpha \neq 1
$$

Hill entropy has the following property:

$$
\begin{gathered}
N_{\alpha}(A+B)=N_{\alpha}(A)+N_{\alpha}(B)+(1-\alpha) \cdot N_{\alpha}(A) \\
\cdot B_{\alpha}(B)
\end{gathered}
$$

Let $p_{1}, p_{2}, \ldots, p_{t}, p_{t+1}, \ldots, p_{k}$ be its "probability distribution of an image with $k$ gray-levels", where $p_{t}$ is the normalized histogram (i.e., $\left.p_{t}=h_{t} /(M \times N)\right)$ and $h_{t}$ is the gray level histogram. Using these probability distributions "one for the object (class A) and the other for the background (class B)" can be derived as:

$$
\begin{gathered}
p_{A}: \frac{p_{1}}{P_{A}}, \frac{p_{2}}{P_{A}}, \ldots, \frac{p_{t}}{P_{A}}, p_{B}: \frac{p_{t+1}}{P_{B}}, \frac{p_{t+2}}{P_{B}}, \ldots, \frac{p_{k}}{P_{B}}, \\
P_{A}=\sum_{i=1}^{t} p_{i}, \quad P_{B}=\sum_{i=t+1}^{k} p_{i}
\end{gathered}
$$

where, $t$ is the threshold value.

"Entropy of object $\left(N_{\alpha}^{A}\right)$ and background $\left(N_{\alpha}^{B}\right)$ pixels" can be calculated as:

$$
N_{\alpha}^{A}=\sum_{i=1}^{W}\left(\frac{p_{i}^{\alpha}}{P_{A}}\right)^{\frac{1}{1-\alpha}}, N_{\alpha}^{B}=\sum_{i=1}^{W}\left(\frac{p_{i}^{\alpha}}{P_{B}}\right)^{\frac{1}{1-\alpha}}
$$

With the help of computed entropy," optimum threshold parameter value" can be obtained as the following:

$$
\begin{gathered}
t^{o p t}=\operatorname{Arg} \max \left[N_{\alpha}^{A}(t)+N_{\alpha}^{B}(t)+(1-\alpha) \cdot N_{\alpha}^{A}(t)\right. \\
\left.\cdot N_{\alpha}^{B}(t)\right]
\end{gathered}
$$

In "multi-level fuzzy Hill entropy", a conventional set $A$ can be expressed as "a group of elements that either belong to or not belongs to set A". While as per the generalized version of fuzzy set, an element can partially belong to a set $A$. Above expression can be shown as:

$$
A=\left\{\left(\mathrm{x}, \mu_{\mathrm{A}}(\mathrm{x}) \mid \mathrm{x} \in \mathrm{X}\right)\right\}
$$

where, $0 \leq \mu_{\mathrm{A}}(\mathrm{x}) \leq 1$ and $\mu_{\mathrm{A}}(\mathrm{x})$ is known as "membership function", which computes the "similarity of $\mathrm{x}$ to A". For simplicity "trapezoidal membership function" is employed to compute the membership of $\mathrm{n}$ segmented regions, $\mu_{1}, \mu_{2}, \ldots, \mu_{\mathrm{n}}$ by using $2 \times(\mathrm{n}-1)$ unknown fuzzy parameters, namely " $\mathrm{a}_{1}, \mathrm{c}_{1}, \ldots \mathrm{a}_{\mathrm{n}-1}, \mathrm{c}_{\mathrm{n}-1}$ where $0 \leq \mathrm{a}_{1} \leq \mathrm{c}_{1} \leq \cdots \leq$ $\mathrm{a}_{\mathrm{n}-1} \leq \mathrm{c}_{\mathrm{n}-1} \leq \mathrm{L}-1$ ”. Subsequently, for $n$ level threshold, "membership function" can be derived as follows.

$$
\begin{aligned}
& \mu_{1}(k)=\left\{\begin{array}{cc}
1 & k \leq a_{1} \\
\frac{k-c_{1}}{a_{1}-c_{1}} a_{1} & \leq k \leq c_{1} \\
0 & k>c_{1}
\end{array}\right. \\
& \mu_{n-1}(k)=\left\{\begin{array}{cc}
0 & k \leq a_{n-2} \\
\frac{k-a_{n-2}}{c_{n-2}-a_{n-2}} a_{n-2} \leq k \leq c_{n-2} \\
\frac{k-c_{n-1}}{a_{n-2}} a_{n-1} \leq k \leq a_{n-2} \\
0 \quad k>c_{n-1} \\
0 \quad k-c_{n-1}
\end{array}\right. \\
& \mu_{n}(k)=\left\{\begin{array}{cc}
1 & k \leq a_{n-1} \\
\frac{k-a_{n}}{c_{n}-a_{n}} a_{n-1} & \leq k \leq c_{n-1} \\
1 & k>c_{n-1}
\end{array}\right.
\end{aligned}
$$

"The maximum fuzzy Hill entropy for each segment of $n-$ level segments" can be expressed by:

$$
\begin{gathered}
\mathrm{H}_{1}^{\alpha}(\mathrm{p})=\left(\sum_{\mathrm{i}=0}^{\mathrm{t}_{1}}\left(\frac{p_{i}}{P_{1}}\right)^{\alpha}\right)^{\frac{1}{1-\alpha}} \\
\mathrm{H}_{2}^{\alpha}(\mathrm{p})=\left(\sum_{\mathrm{i}=\mathrm{t} 1+1}^{\mathrm{t}_{\mathrm{n}}}\left(\frac{p_{i}}{P_{n}}\right)^{\alpha}\right)^{\frac{1}{1-\alpha}} \\
\mathrm{H}_{\mathrm{n}}^{\alpha}(\mathrm{p})=\frac{1}{1-\alpha}\left(\sum_{\mathrm{i}=\mathrm{t}_{\mathrm{n}-1}+1}^{\mathrm{L}-1}\left(\frac{p_{i}}{P_{n}}\right)^{\alpha}\right)^{\frac{1}{1-\alpha}}
\end{gathered}
$$

where, $\quad \alpha \neq 1, \quad P_{1}=\sum_{i=0}^{\mathrm{t}_{1}} p_{i} * \mu_{1}(i), P_{2}=\sum_{i=0}^{\mathrm{t}_{2}} p_{i} *$ $\mu_{2}(i), \cdots, P_{n}=\sum_{i=0}^{L-1} p_{i} * \mu_{n}(i)$.

"The optimum value of parameters" can be obtained by maximizing the total entropy.

$$
\begin{aligned}
\varphi\left(a_{1}, c_{1}, \ldots a_{n-1},\right. & \left.c_{n-1}\right) \\
& =\operatorname{ArgMax}\left(\left[H_{1}(t)+H_{2}(t) \ldots\right.\right. \\
& \left.\left.+H_{n}(t)\right]\right)
\end{aligned}
$$

"The (n-1) threshold values can be obtained by employing the fuzzy parameters" as:

$$
\begin{aligned}
t_{1}=\frac{\left(a_{1}+c_{1}\right)}{2}, t_{2} & =\frac{\left(a_{2}+c_{2}\right)}{2}, \ldots, t_{n-1} \\
& =\frac{\left(a_{n-1}+c_{n-1}\right)}{2}
\end{aligned}
$$

In the proposed approach, we utilize Differential Evolution (DE) [19] which is a population-based global optimization technique Hill entropy is optimized to acquire an image threshold by Differential Evolution "DE" optimization algorithm. In the following an overview of differential evolution:

An individual parameter vector $\left(i^{t h}\right)$ of the population at generation time $(t)$ in $D$-dimensional space vector can be expressed as:

$$
\overrightarrow{Z_{l}}(t)=\left[Z_{i, 1}(t), Z_{i, 2}(t), \ldots, Z_{i, D}(t)\right]
$$


The donor vector $\vec{Y}_{l}(t)$ is constructed in every generation to modify the population members $\overrightarrow{Z_{l}}(t)$. Three different parameter vectors are collected at random from the current population " $\left(r_{1}, r_{2}\right.$ and $r_{3}$-th vectors such that $r_{1}, r_{2}, r_{3} \in$ $[1, N P]$ and $r_{1} \neq r_{2} \neq r_{3}$ ) "to construct the donor vector $\vec{Y}_{l}(t)$ for every $i^{t h}$ member in one of earliest variants of $\mathrm{DE}$. The donor vector $\vec{Y}_{l}(t)$ is acquired by "multiplying a scalar number $F$ with the difference of any two of three other parameter vectors". The procedure for the $j^{t h}$ component of the $i^{t h}$ vector can be articulated like:

$$
\overrightarrow{Y_{l, j}}(t)=\left[Z_{r_{1}, j}(t)+F \cdot\left(Z_{r_{2}, j}(t)-Z_{r_{3}, j}(t)\right]\right.
$$

"A binomial crossover operation" is performed to enhance the possible diversity of the population and achieved on every one of the $D$ variables when a randomly selected number between 0 and 1 is within the $C_{r}$ value. In this case, the binomial distribution is followed by the number of parameters derived from the mutant. Therefore, each target vector $\overrightarrow{Z_{l}}(t)$ and the trial vector $\overrightarrow{R_{l}}(\mathrm{t})$ are constructed as follows:

$$
R_{i, j}(t)=\left\{\begin{array}{l}
Y_{i, j}(t) \text { if } \operatorname{rand}_{j}(0,1) \leq C_{r} \text { or } j=r n(i) \\
Z_{i, j}(t) \text { if } \operatorname{rand}_{j}(0,1)>C_{r} \text { or } j \neq r n(i)
\end{array}\right.
$$

The above equation is applicable for $j=1,2, \ldots$ Parameter $\operatorname{rand}_{j}(0,1) \in[0,1]$ is the $j^{\text {th }}$ evaluation of a uniform random number generator $r n(i) \in[1,2, \ldots, D]$. It is a randomly chosen index to guaranteed that $\overrightarrow{R_{l}}(t)$ gets at least one component from $\overrightarrow{Z_{l}}(t)$. In the last step, a selection method is employed to enhance the results. In case of the "cost function of the target vector is greater than the trial vector, the trial vector is replaced by the target vector on the subsequent generation", otherwise; the target vector will continue in the population i.e.

$$
\overrightarrow{Z_{l}}(t+1)=\left\{\begin{array}{l}
\overrightarrow{R_{l}}(t) \text { if } f\left(\overrightarrow{R_{l}}(t)\right)>f\left(\left(\overrightarrow{Z_{l}}(t)\right)\right. \\
\overrightarrow{Z_{l}}(t) \text { if } f\left(\overrightarrow{R_{l}}(t)\right) \leq f\left(\overrightarrow{Z_{l}}(t)\right)
\end{array}\right.
$$

\section{EXPERIMENTAL RESULTS}

The proposed technique has been tested on different low contrast medical images. The simulations have been performed with MATLAB R2018a. We used sample of dataset of medical images shown in Figure 1. The goal is to efficiently segment an image in three different levels. Note that "for $n$ levels segmentation problem the dimensionality of the search space is $D=2 *(n-1)$ as number of unknown fuzzy parameters are 2". To verify the efficiency of the proposed segmentation approach, we compare our method with Tao et al. [21] and Tang [22]. Experiments have been carried using low contrast medical images. In this section various types of dataset, including images of human brain, Lung and Liver have been employed. In our experimental, "we first present the comparison results of the proposed approach using different three level of threshold". Subsequently, the results for Tao et al. [21] and Tang [22] have been provided. Finally, Table 1 has been given to show the "details of parameters obtained in the experiments of the proposed approach".

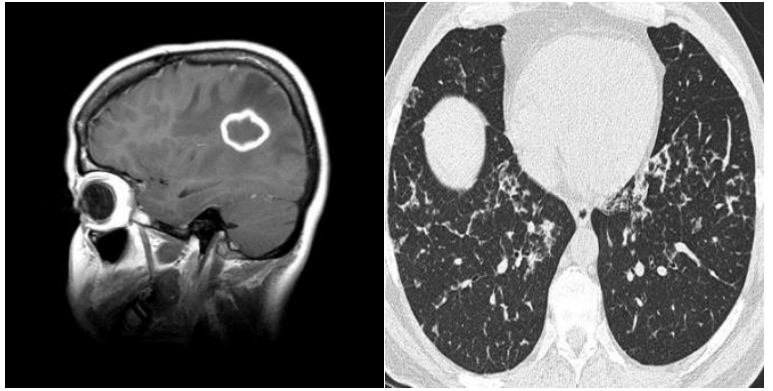

(a)

(b)

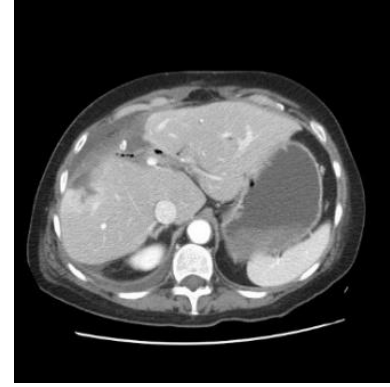

(c)

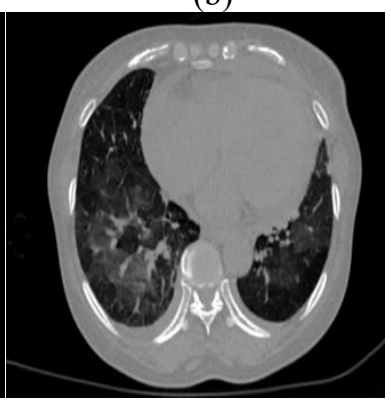

(d)
Figure 1. Sample of medical images

We have compared our method with similar existing techniques based on qualitative evaluation. For that, the first original image of human brain and their corresponding qualitative results have been shown in Figure 2 (a), where level 2 , level 3, and level 4 of threshold-based segmentation results of our approach have been shown in Figure 2 (b), (c), and (d) respectively. The experimental results for Tao et al, method [21] and Tang method [22] have been shown in Figure 2 (e) and (f) respectively. It has been clearly visible from the results obtained through our approach make the cerebral cortex outstanding i.e., perform better segmentation than other techniques. In the similar way, Lung image's results have been shown in Figure 3, where original image has been shown in Figure 3 (a), level 2, level 3, and level 4 of threshold-based segmentation results for our proposed technique have been shown in Figure 3 (b), (c), and (d) respectively. The results of Tao et al., method [21] and Tang method [22] have been shown in Figure 3 (e) and (f) respectively. Again, it has been evident from the results that segmentation performance of our technique is far better than compared approaches. The results with the third image "Liver" have been shown in Figure 4, where original image has been shown in Figure 4 (a), and level 2 level 3, and level 4 threshold-based segmentation results of our approach have been shown in Figure 4 (b), (c), and (d) respectively. Tao et al., [21] and Tang [22] results on Liver image have been displayed in figure 4(e) and (f) respectively. The results with the fourth image have been shown in Figure 5 , where original image has been shown in Figure 5 (a), and level 2 level 3, and level 4 threshold-based segmentation results of our approach have been shown in Figure 5 (b), (c), and (d) respectively. Tao et al., [21] and Tang [22] results on Liver image have been displayed in Figure 5(e) and (f) respectively. It is evident from experiment results and qualitative performance analysis that our algorithm outperforms existing methods especially in case of low contrast image. 


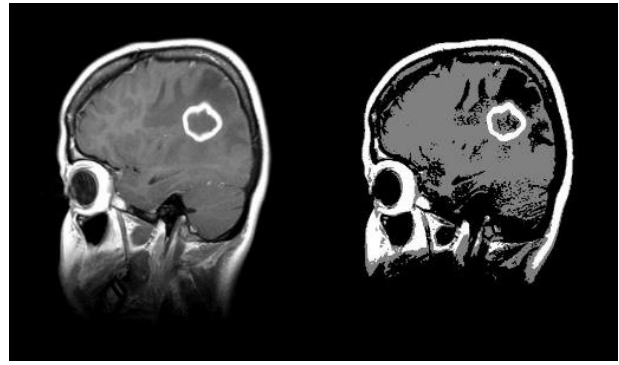

(a)

(b)

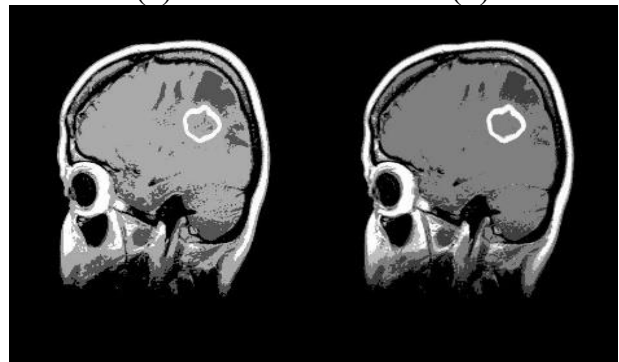

(c)

(d)

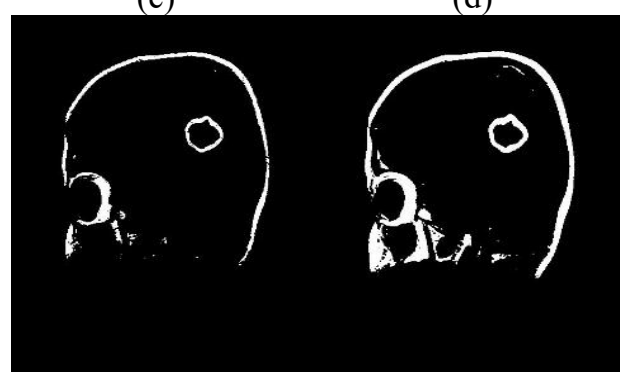

(e) Tang method [22] (f) Tao Method [21]

Figure 2. Results with human brain image
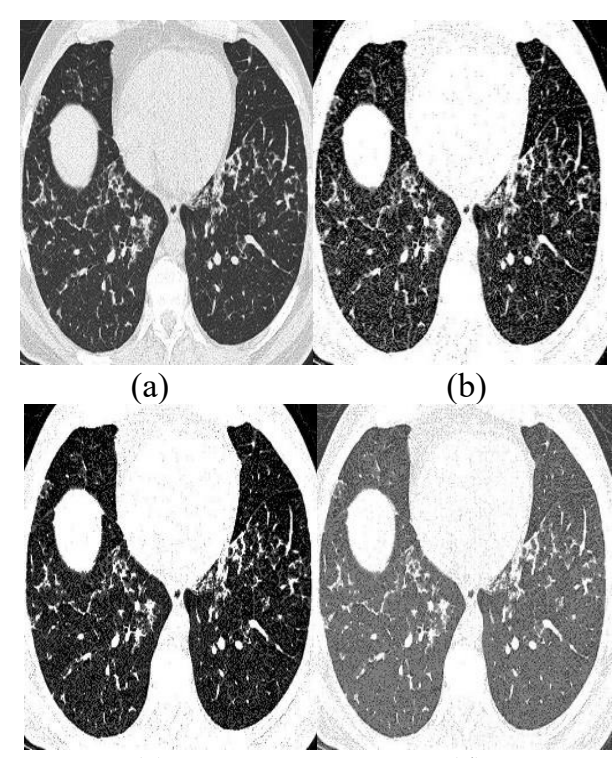

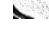

(c)

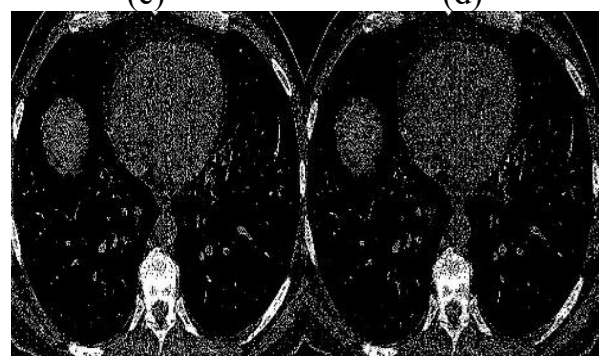

(e) Tang method [22] (f) Tao Method [21]

Figure 3. Results with lung image

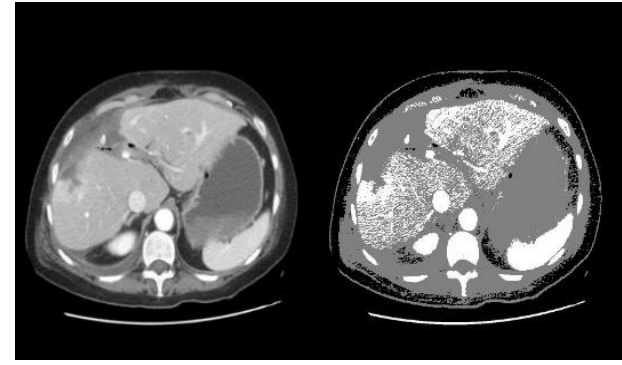

(a)

(b)

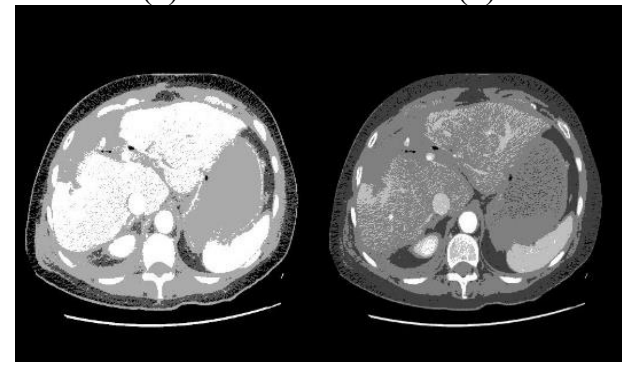

(c)

(d)

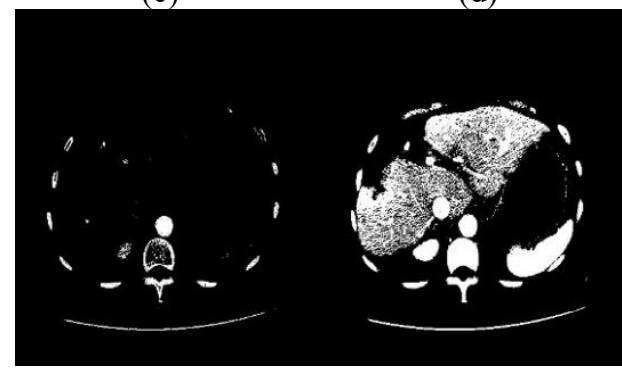

(e) Tang method [22] (f) Tao Method [21]

Figure 4. Results with liver image

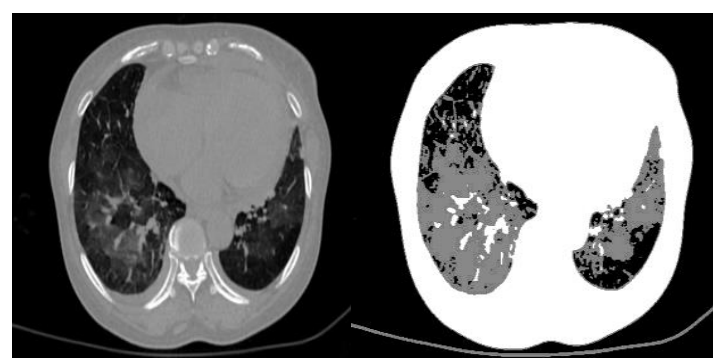

(a)

(b)

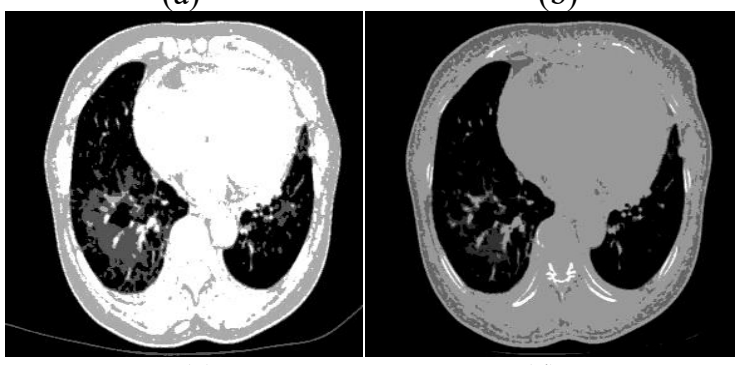

(c)

(d)

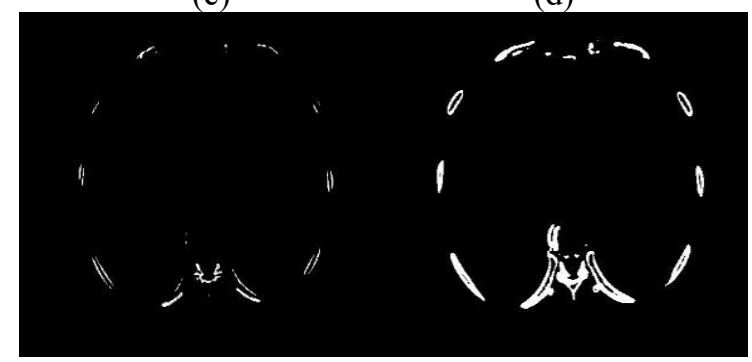

(e) Tang method [22] $\quad$ (f) Tao method [21]

Figure 5. Results with lung COVID 19 image 
Table 1. Parameter values obtained using DE for 2-4 level thresholds of the proposed approach

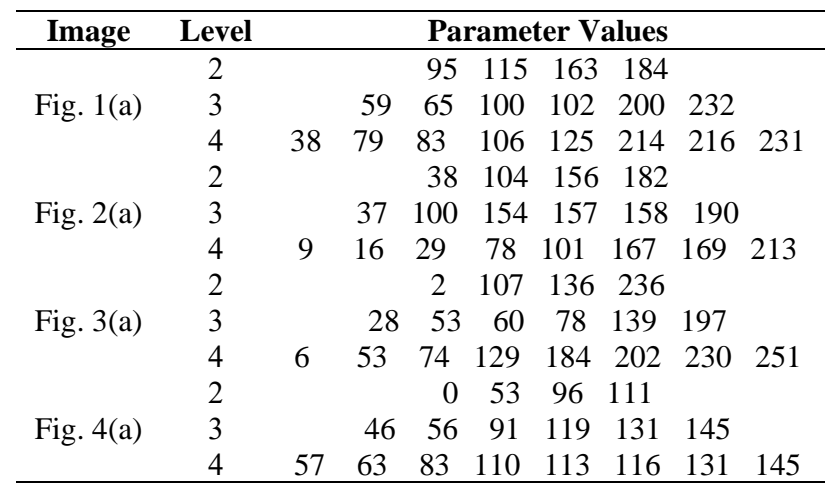

\section{CONCLUSIONS}

Different methods for medical image segmentation have been analyzed and found that segmentation accuracy for low contrast image is not significant. Therefore, Differential evolution optimization-based Hill entropy for low contrast medical image segmentation is proposed. Our approach is superior to others because it considers not only the information of background and foreground, but also have role of foreground and background in threshold computation process. Another advantage of our technique is that after threshold, it preserves main features from original image. This technique has improvement than existing approaches in terms of "increased accuracy and reduced computational complexity". The performance evaluation and experimental results proves that the proposed technique can effectively handle segmentation task especially in case of low contrast image.

\section{REFERENCES}

[1] Salem, M.A.M., Atef, A., Salah, A., Shams, M. (2017). Recent survey on medical image segmentation. In Handbook of Research on Machine Learning Innovations and Trends, 424-464. https://doi.org/10.4018/978-15225-2229-4.ch019

[2] Kumar, S.A., Harish, B.S. (2018). A modified intuitionistic fuzzy clustering algorithm for medical image segmentation. Journal of Intelligent Systems, 27(4): 593-607. https://doi.org/10.1515/jisys-2016-0241

[3] Elaraby, A., Nechaevskiy, A. (2021). An effective segmentation approach for liver computed tomography scans using fuzzy exponential entropy. Computer Research and Modeling, 13(1): 195-202. https://doi.org/10.20537/2076-7633-2021-13-1-195-202

[4] Zhao, M., Fu, A.M., Yan, H. (2001). A technique of three-level thresholding based on probability partition and fuzzy 3-partition. IEEE Transactions on Fuzzy Systems, $9(3)$ :

469-479. https://doi.org/10.1109/91.928743

[5] Tao, W.B., Tian, J.W., Liu, J. (2003). Image segmentation by three-level thresholding based on maximum fuzzy entropy and genetic algorithm. Pattern Recognition Letters, 24(16): 3069-3078. https://doi.org/10.1016/S0167-8655(03)00166-1

[6] Kapur, J.N., Sahoo, P.K., Wong, A.K. (1985). A new method for gray-level picture thresholding using the entropy of the histogram. Computer Vision, Graphics, and Image Processing, 29(3): 273-285. https://doi.org/10.1016/0734-189X(85)90125-2

[7] Abutaleb, A.S. (1989). Automatic thresholding of graylevel pictures using two-dimensional entropy. Computer Vision, Graphics, and Image Processing, 47(1): 22-32. https://doi.org/10.1016/0734-189X(89)90051-0

[8] Zennouhi, R., Masmoudi, L.H. (2009). A new 2D histogram scheme for colour image segmentation. The Imaging Science Journal, 57(5): 260-265. https://doi.org/10.1179/136821909X12490307952874

[9] Sengar, S.S., Mukhopadhyay, S. (2016). Moving object area detection using normalized self adaptive optical flow. Optik, 127(16): 6258-6267. https://doi.org/10.1016/j.ijleo.2016.03.061

[10] Sengar, S.S., Mukhopadhyay, S. (2017). Detection of moving objects based on enhancement of optical flow. Optik, 145: 130-141. https://doi.org/10.1016/j.ijleo.2017.07.040

[11] Sengar, S.S., Mukhopadhyay, S. (2017). Motion detection using block based bi-directional optical flow method. Journal of Visual Communication and Image Representation, 49: 89-103. https://doi.org/10.1016/j.jvcir.2017.08.007

[12] Cheng, H.D., Chen, C.H., Chiu, H.H., Xu, H. (1998). Fuzzy homogeneity approach to multilevel thresholding. IEEE Transactions on Image Processing, 7(7): 10841086. https://doi.org/10.1109/83.701171

[13] El-Feghi, I., Galhoud, M., Sid-Aadhmed, M.A., Ahmadi, M. (2007). Three-level gray-scale images segmentation using non-extensive entropy. Computer Graphics, Imaging and Visualisation (CGIV 2007), pp. 304-307. https://doi.org/10.1109/CGIV.2007.83

[14] Chen, X., Williams, B.M., Vallabhaneni, S.R., Czanner, G., Williams, R., Zheng, Y. (2019). Learning active contour models for medical image segmentation. 2019 IEEE/CVF Conference on Computer Vision and Pattern Recognition (CVPR), pp. 11624-11632. https://doi.org/10.1109/CVPR.2019.01190

[15] Jégou, S., Drozdzal, M., Vazquez, D., Romero, A., Bengio, Y. (2017). The one hundred layers tiramisu: Fully convolutional DenseNets for semantic segmentation. 2017 IEEE Conference on Computer Vision and Pattern Recognition Workshops (CVPRW), pp. $1175-1183$ https://doi.org/10.1109/CVPRW.2017.156

[16] Ronneberger, O., Fischer, P., Brox, T. (2015). U-net: Convolutional networks for biomedical image segmentation. In International Conference on Medical Image Computing and Computer-Assisted Intervention, pp. 234-241. https://doi.org/10.1007/978-3-319-245744_28

[17] Vese, L.A., Chan, T.F. (2002). A multiphase level set framework for image segmentation using the Mumford and Shah model. International Journal of Computer Vision, 50(3): 271-293. https://doi.org/10.1023/A:1020874308076

[18] Elaraby, A., Moratal, D. (2017). A generalized entropybased two-phase threshold algorithm for noisy medical image edge detection. Scientia Iranica, 24(6): 3247-3256. https://doi.org/10.24200/SCI.2017.4359

[19] Opara, K.R., Arabas, J. (2019). Differential evolution: A survey of theoretical analyses. Swarm and Evolutionary Computation, 44: 546-558. 
https://doi.org/10.1016/j.swevo.2018.06.010

[20] Gray, R.M. (2013). Entropy and Information Theory. Springer. https://doi.org/10.1007/978-1-4419-7970-4

[21] Tao, W., Jin, H., Liu, L. (2007). Object segmentation using ant colony optimization algorithm and fuzzy entropy. Pattern Recognition Letters, 28(7): 788-796. https://doi.org/10.1016/j.patrec.2006.11.007

[22] Tang, Y., Di, Q., Guan, X., Liu, F. (2008). Threshold selection based on fuzzy tsallis entropy and particle swarm optimization. NeuroQuantology, 6(4): 412-419. https://doi.org/10.14704/NQ.2008.6.4.197 